\title{
Investigação em programação com Scratch para crianças: uma revisão sistemática da literatura
}

\author{
Saymon S. Souza, Thais H. C. Castro \\ Instituto de Computação - Universidade Federal do Amazonas (UFAM) \\ Av. Rodrigo Otávio, 3000 - 69.080-900 - Manaus - AM - Brasil \\ \{sss, thais\} @icomp.ufam.edu.br
}

\begin{abstract}
Studies done by well-known national and international institutions reveal the importance of teaching programming from basic education to the formation of professionals in the IT area, but there are many obstacles to the implementation of this idea. In this context, this article aims to present a systematic review of articles on the use of Scratch tool for teaching programming for children, published in the last nine years (2007-2016) and presented in major national and international events. The results showed that researches focus on methodologies for teaching and that these have brought positive results when applied in practical context.
\end{abstract}

Resumo. Estudos feitos por renomadas instituições nacionais e internacionais revelam a importância do ensino de programação desde a educação básica para a formação de profissionais na área de TI, porém existem muitos obstaculos para a implementação desta ideia. Nesse contexto, este artigo tem como objetivo apresentar uma revisão sistemática dos artigos referentes à utilização da ferramenta Scratch para o ensino de programação para crianças, publicados nos anos nove anos (2007-2016) e apresentado nos principais eventos nacionais e internacionais. Os resultados obtidos mostraram que as pesquisas focam em metodologias para o ensino e que essas trouxeram resultados positivos quando aplicadas no contexto prático.

\section{Introdução}

São muitas as dificuldades encontradas pelos alunos durante o processo de ensino ou aprendizagem de programação. A citar algumas, a dificuldade de aprendizado, dificuldade por parte do professor para apresentar o conteúdo, a grande exigência de conhecimento de lógica e matemática e o contato tárdio com conceitos de lógica de programação.

Nesse contexto, o ensino de conceitos de programação ainda na educação básica pode ser um grande facilitador para o processo de aprendizagem. Várias iniciativas vem sendo tomadas para que o pensamento computacional e o raciocínio lógico sejam desenvolvidos desde cedo, uma delas é o uso de ferramentas para o auxílio do ensino desses conceitos. A ferramenta mais utilizada para o ensino e pesquisa atualmente é o Scratch ${ }^{1}$. 
V Congresso Brasileiro de Informática na Educação (CBIE 2016)

Anais dos Workshops do V Congresso Brasileiro de Informática na Educação (CBIE 2016)

Scratch é uma ferramenta criada em 2009 pelo Prof. Dr. Mitchel Resnick do Instituto de Tecnologia de Massachusetts (MIT) que tem como objetivo ensinar conceitos de programação para pessoas de idade entre 8 e 16 anos utilizando blocos lógicos, itens de imagem e som para o desenvolvimento de histórias interativas, animações e jogos, além de possibilidade de compartilhar online essas criações para todos.

Atualmente, inúmeras pesquisas vem se aproveitando do potencial de ensino e aprendizagem que a ferramenta Scratch possui para aplicá-la em sala de aula com crianças da educação básica. Nesse contexto, há uma necessidade clara de se analisar a produção cientifíca existente sobre o uso dessa ferramenta para o ensino e aprendizagem de programação. Para que tal objetivo fosse alcançado, foi realizada uma Revisão Sistemática da Literatura (RSL) que contou com a análise de artigos sobre o processo de ensino e aprendizagem de conceitos de programação com a utilização da ferramenta Scratch publicados nos últimos 9 anos (2007-2016) em eventos importantes nacionais e internacionais. Cada artigo foi analisado utilizando as escalas sistemáticas definidas pela escala PEDro ${ }^{2}$, que será apresentada na seção 2 deste artigo.

O objetivo deste artigo é mostrar os resultados obtidos na RSL e fazer uma análise crítica do mesmo, mostrando tanto o que a acadêmia vem produzindo na área de ensino de programação para crianças usando Scratch, como oportunidades de trabalhos futuros.

Este artigo está organizado da seguinte forma. A seção 2 apresenta a fundamentação téorica por trás dos metódos utilizados neste artigo e serve como base para a explanação dos dados obtidos. Na seção 3 são apresentados os resultados obtidos na RSL. Já na seção 4 é feita a análise dos resultados e, por fim, na seção 5 são descritas as oportunidades para trabalhos futuros.

\section{Métodos Utilizados}

Uma Revisão Sistemática da Literatura (RSL) é um estudo secundário que tem como objetivo reunir estudos semelhantes, avaliando-os criticamente em comparação a características como a fonte de pesquisa, questão do estudo, infererências, avaliação, seleção de publicações, entre outras e os reunindo em uma análise estátistica.

A escala PEDro foi desenvolvida pela Physiotherapy Evidence Database com o objetivo de auxiliar pesquisadores a identificar de forma rápida quais estudos de uma base de dados podem conter informação estátistica suficiente para que os resultados obtidos possam ser interpretados. Cada artigo recebe uma pontuação de avaliação da qualidade que varia de 0 a 10 , sendo considerada de qualidade alta artigos com pontuações acima de 6. A utlização da escala PEDro para avaliação dos dados obtidos será discutida na seção 2.3 , onde será detalhada a forma de avaliação usada pela escala e como ela foi utilizada para classificar os trabalhos estudados durante a RSL.

$1<$ http://scratch.mit.edu>

$2<$ http://www.pedro.org.au/portuguese/downloads/pedro-scale> 
V Congresso Brasileiro de Informática na Educação (CBIE 2016)

Anais dos Workshops do V Congresso Brasileiro de Informática na Educação (CBIE 2016)

\subsection{Questões da Pesquisa}

A RSL teve como questão central a seguinte pergunta: Qual é a panoroma atual da utilização de Scratch no ensino de programação para crianças? Para encontrar a resposta a essa pergunta, as segunites questões foram definidas:

- QE1: Quais foram as abordagens utilizadas para o uso da ferramenta em sala de aula?

- QE2: Quais foram os resultados obtidos ao utilizar a ferramenta em sala de aula?

- QE3: Quais são as instituições de pesquisa envolvidas na área?

- QE4: Quais foram os efeitos observados nos alunos com a introdução de atividades de programação baseadas em Scratch?

\subsection{Fontes de busca, processo de seleção e critérios de inclusão e exclusão}

A pesquisa foi realizada por meio de uma busca manual nas bases de dados Scopus, ACM e DERWENT-Períodicos Capes. A busca foi dividida em duas etapas. Na primeira etapa foi realizada uma pré-seleção com base nos artigos encontrados nas bases de dados supracitados e lendo os seus títulos e abstracts. Na segunda etapa, todos os artigos pré-selecionados foram analisados e os critérios de exclusão e exclusão apresentados na Tabela 1 foram aplicados a cada um deles.

Tabela 1. Critérios de inclusão e exclusão

\begin{tabular}{|c|c|}
\hline Critérios de Inclusão & Critérios de Exclusão \\
\hline $\begin{array}{c}\text { Artigos que tenham usado Scratch em seus } \\
\text { experimentos. } \\
\text { Artigos que tenham mostrado métricas } \\
\text { sobre a utilização da ferramenta. } \\
\text { Artigos que tenham sido publicados a } \\
\text { partir } 2007 . \\
\text { Artigos que tenham usado ferramentas de } \\
\text { apoio a Scratch. }\end{array}$ & $\begin{array}{l}\text { Artigos que não tenham utilizado Scratch. } \\
\text { Artigos que com experimentos feitos com } \\
\text { pessoas com idades menores do que 9anos } \\
\text { e maiores do que } 12 \text { anos. }\end{array}$ \\
\hline
\end{tabular}

\subsection{Avaliação da Qualidade}

A escala PEDro foi utilizada para obter uma pontuação final para cada artigo. Para cada um dos critérios da escala PEDro,o procedimento para pontuação é de 'Sim' e 'Não', que equivalem a 1 e 0 pontos respectivamente. Os critérios da escala PEDro utilizados na RSL foram:

- C1: Os critérios de elegibilidade foram especidados;

- C2: Os sujeitos foram aleatoriamente distribuídos por grupo;

- C3: A alocação dos sujeitos foi secreta;

- C4: Inicialmente, os grupos eram semelhantes no que diz respeito aos indicadores de prognóstico mais importantes; 
V Congresso Brasileiro de Informática na Educação (CBIE 2016)

Anais dos Workshops do V Congresso Brasileiro de Informática na Educação (CBIE 2016)

- C5: Todos os sujeitos participaram de forma cega no estudo;

- C6: Todos os avaliadores que mediram pelo menos um resultado-chave, fizeram-no de forma cega;

- C7: Mensurações de pelo menos um resultado-chave foram obtidas em mais de $85 \%$ dos sujeitos inicialmente distribuídos pelos grupos;

- C8: Os resultados das comparações inter-grupos foram descritos para pelo menos um resultado chave;

- C9: O estudo apresenta tanto medidas de precisão como medidas de variabilidade para pelo menos um resultado-chave.

Os critérios mostrados a seguir não foram utilizados por não se adequar a proposta da RSL:

- C10: Todos os terapeutas que administraram a terapia fizeram-no de forma cega;

- C11: Todos os sujeitos a partir dos quais se apresentaram mensurações de resultados receberam o tratamento ou a condição de controle conforme a alocação, ou, quando não foi esse o caso, fez-se a análise dos dados para pelo menos um dos resultados-chave por "intenção de tratamento";

A pontuação na escala PEDro necessária para que um artigo fosse incluído na RSL era de 6.

\section{Resultados}

A busca resultou em 32 artigos, dentre eles, 12 foram incluídos. A tabela 2 apresenta $o$ resultado geral da busca. Para encontrar os 32 artigos pré-selecionados, a seleção se deu pela leitura dos títulos e abstracts.

Tabela 2. Resultado geral da busca.

\begin{tabular}{|c|c|c|c|}
\hline Base de Dados & Total de Artigos & $\begin{array}{c}\text { Artigos Pré- } \\
\text { Selecionados }\end{array}$ & Artigos Incluídos \\
\hline ACM & 31 & 18 & 5 \\
\hline Scopus & 15 & 4 & 2 \\
\hline $\begin{array}{c}\text { DERWENT - } \\
\text { Períodicos } \\
\text { Capes }\end{array}$ & 22 & 10 & 5 \\
\hline TOTAL & $\mathbf{6 8}$ & $\mathbf{3 2}$ & $\mathbf{1 2}$ \\
\hline
\end{tabular}

Em seguida, foi realizada uma leitura completa dos artigos pré-selecionados e uma verificação para saber se os mesmos estavam de acordo com os critérios de inclusão e excluão. Após essa etapa, 12 artigos se mostraram relevantes para a pesquisa e foram incluídos na RSL. A tabela 3 lista os artigos incluídos e as suas respectivas pontuações na escala PEDro de acordo com os critérios e métricas apresentados na seção 2.3 deste artigo. 
V Congresso Brasileiro de Informática na Educação (CBIE 2016)

Anais dos Workshops do V Congresso Brasileiro de Informática na Educação (CBIE 2016)

Tabela 3. Artigos Incluídos

\begin{tabular}{|c|c|c|c|c|}
\hline ID & Título & Universidade & $\begin{array}{c}\text { Base de } \\
\text { dados }\end{array}$ & $\begin{array}{l}\text { Escala PEDRo } \\
\text { - Pontuação }\end{array}$ \\
\hline 1 & $\begin{array}{l}\text { Visual } \\
\text { programming } \\
\text { languages } \\
\text { integrated across } \\
\text { the curriculum in } \\
\text { elementary school: } \\
\text { A two year case } \\
\text { study using } \\
\text { "Scratch" in five } \\
\text { schools. }\end{array}$ & $\begin{array}{c}\text { Spanish } \\
\text { National } \\
\text { University of } \\
\text { Distance } \\
\text { Education - } \\
\text { UNED - } \\
\text { Espanha }\end{array}$ & $\begin{array}{c}\text { DERWENT } \\
\text { - Periódicos } \\
\text { CAPES }\end{array}$ & 9 \\
\hline 2 & $\begin{array}{l}\text { Programming by } \\
\text { Choice: Urban } \\
\text { Youth Learning } \\
\text { Programming with } \\
\text { Scratch }\end{array}$ & $\begin{array}{l}\text { University of } \\
\text { California - } \\
\text { Los Angeles } \\
\text { - EUA }\end{array}$ & Scopus & 7 \\
\hline 3 & $\begin{array}{l}\text { An investigation of } \\
\text { the effects of } \\
\text { programming with } \\
\text { Scratch on the } \\
\text { preservice IT } \\
\text { teachers' self- } \\
\text { efficacy } \\
\text { perceptions and } \\
\text { attitudes towards } \\
\text { computer } \\
\text { programming }\end{array}$ & $\begin{array}{c}\text { Kirkkale } \\
\text { University - } \\
\text { Turquia }\end{array}$ & $\begin{array}{c}\text { DERWENT } \\
\text { - Periódicos } \\
\text { CAPES }\end{array}$ & 7 \\
\hline 4 & $\begin{array}{c}\text { Ensino de } \\
\text { Computação com } \\
\text { Scratch no Ensino } \\
\text { Fundamental - Um } \\
\text { estudo de caso }\end{array}$ & $\begin{array}{c}\text { Universidade } \\
\text { Federal de } \\
\text { Santa } \\
\text { Catarina } \\
\text { (UFSC) - } \\
\text { Brasil } \\
\end{array}$ & $\begin{array}{c}\text { DERWENT } \\
\text { - Periódicos } \\
\text { CAPES }\end{array}$ & 9 \\
\hline 5 & $\begin{array}{c}\text { Teaching } \\
\text { Computer Science } \\
\text { to Young Children } \\
\text { through creativity: } \\
\text { Lessons learned } \\
\text { from the case of } \\
\text { Norway }\end{array}$ & $\begin{array}{l}\text { Norwegian } \\
\text { University of } \\
\text { Science and } \\
\text { Technology } \\
\text { - Noruega }\end{array}$ & $\mathrm{ACM}$ & 9 \\
\hline 6 & $\begin{array}{l}\text { The Effects of } \\
\text { Teaching } \\
\text { Programming via }\end{array}$ & $\begin{array}{c}\text { Baskent } \\
\text { University - } \\
\text { Ankara } \\
\end{array}$ & $\mathrm{ACM}$ & 9 \\
\hline
\end{tabular}


V Congresso Brasileiro de Informática na Educação (CBIE 2016)

Anais dos Workshops do V Congresso Brasileiro de Informática na Educação (CBIE 2016)

\begin{tabular}{|c|c|c|c|c|}
\hline & $\begin{array}{c}\text { Scratch on } \\
\text { Problem Solving } \\
\text { Skills: A } \\
\text { Discussion from } \\
\text { Learners' } \\
\text { Perspective } \\
\end{array}$ & $\begin{array}{l}\text { University - } \\
\text { Turquia }\end{array}$ & & \\
\hline 7 & $\begin{array}{l}\text { The Benefits of } \\
\text { Using Scratch to } \\
\text { Introduce Basic } \\
\text { Programming } \\
\text { Concepts in the } \\
\text { Elementary } \\
\text { Classroom }\end{array}$ & $\begin{array}{l}\text { The Fraser- } \\
\text { Woods } \\
\text { School - } \\
\text { Inglaterra }\end{array}$ & Scopus & 6 \\
\hline 8 & $\begin{array}{l}\text { Skill Progression } \\
\text { in Scratch } \\
\text { Revisited }\end{array}$ & $\begin{array}{l}\text { MIT Media } \\
\text { Lab - EUA }\end{array}$ & $\begin{array}{c}\text { DERWENT } \\
\text { - Periódicos } \\
\text { CAPES }\end{array}$ & 8 \\
\hline 9 & $\begin{array}{l}\text { Embedding } \\
\text { Scratch in the } \\
\text { Classroom }\end{array}$ & $\begin{array}{c}\text { Redware } \\
\text { Research ltd. } \\
\text { - Inglaterra } \\
\end{array}$ & $\mathrm{ACM}$ & 8 \\
\hline 10 & $\begin{array}{l}\text { Learning Complex } \\
\text { Systems with } \\
\text { story-building in } \\
\text { Scratch }\end{array}$ & $\begin{array}{c}\text { Vanderbilt } \\
\text { University - } \\
\text { EUA }\end{array}$ & $\mathrm{ACM}$ & 7 \\
\hline 11 & $\begin{array}{l}\text { Pensamento } \\
\text { Computacional: } \\
\text { transformando } \\
\text { ideias em jogos } \\
\text { digitais usando o } \\
\text { Scratch } \\
\end{array}$ & $\begin{array}{l}\text { Universidade } \\
\text { de São Paulo } \\
\text { (USP) - } \\
\text { Brasil }\end{array}$ & $\begin{array}{c}\text { DERWENT } \\
\text { - Periódicos } \\
\text { CAPES }\end{array}$ & 8 \\
\hline 12 & $\begin{array}{l}\text { Undergraduates } \\
\text { Teach Game } \\
\text { Programming } \\
\text { Using Scratch }\end{array}$ & $\begin{array}{l}\text { University of } \\
\text { Washington } \\
\text { Bothell }\end{array}$ & $\mathrm{ACM}$ & 6 \\
\hline
\end{tabular}

Os artigos que obtiveram pontuação igual a 9 na escala PEDro, a citar os de ID 1/4/5/6, se destacaram diante dos outros por não apenas mostrar a aplicação da ferramenta Scratch no ambiente escolar, como também trazer diferentes possibilidades para a execução de tal atividade. Por trazer algo novo juntamente com a execução das atividades descrita de forma detalhada nos artigos, esses trabalhos mostraram que podem ser rica fonte de informações sobre como a ferramenta Scratch pode ser utilizada de forma produtiva e o que ainda pode se melhorar na utilização do mesmo.

Já os artigos que obtiveram pontuação igual a 6 na escala PEDro, a citar os de ID 7 e 12, mostraram ter conteúdo o suficiente para serem incluídos na RSL por, além de demonstrar a utilização da ferramenta Scratch, fazerem uma análise no impacto que a 
V Congresso Brasileiro de Informática na Educação (CBIE 2016)

Anais dos Workshops do V Congresso Brasileiro de Informática na Educação (CBIE 2016)

ferramenta trouxe para o dia-a-dia dos ambientes em que os experimentos foram realizados. Experiências tais como as mostradas nesses artigos servem pra mostrar a capacidade que a ferramenta Scratch possui para o ensino de conceitos de programação para crianças.

\section{Análises dos resultados}

Após o término da RSL foram identificados alguns pontos fracos e oportunidades a seres exploradas em trabalhos futuros, quanto aos trabalhos futuros, elas serão detalhados na seção 5 deste artigo.

A partir dos resultados obtidos na RSL, observa-se que poucos artigos se preocuparam em fazer um estudo mais aprofundado dos artefatos produzidos após os experimentos. Uma oportunidade perdida visto que a utilização do Scratch em sala de aula traz inúmeras oportunidades para criação de novas metodologias para o ensino da programação. Fugir dos métodos tradicionais e já amplamente praticados poderia trazer maiores ganhos para a comunidade cientifica em geral.

Outro ponto fraco é o problema retratado por vários estudos brasileiros que mostram a dificuldade de disseminar a cultura do ensino de programação no país. Levar tais ideias para todo o país exigiria dar infraestrutura de qualidade e treinamento para professores, algo que em ainda não é uma força em nosso país.

\section{Conclusão e Trabalhos futuros}

Este trabalho objetivou fazer uma prospecção detalhada sobre técnicas, linguagens e cursos de programação para crianças, visando sua aplicabilidade em currículo comuns do ensino fundamental em Ciências e Matemática. Foi confirmada nossa suposição inicial que o Scratch tem sido uma linguagem mais utilizada e apropriada a esse contexto.

Para trabalhos futuros, o objetivo é investigar e mapear o conhecimento em desenvolvimento de habilidades cognitivas de resolução de problemas através da aprendizagem de programação de crianças de 9 a 12 anos, matriculadas no Ensino Fundamental, em escolas de Manaus. Após o mapeamento inicial, pretende-se verificar sua adequabilidade atráves do desenvolvimento de uma ferramenta computacional para anomalias nos registros. O objetivo geral citado acima pode ser atingido através dos seguintes objetivos especifícos:

1. Registrar as interações e os artefatos produzidos nas três aplicações do curso básico de Scratch;

2. Identificar diferenças entre os processos de solução dos bem e mal sucedidos;

3. Desenvolver uma ferramenta para análise semi-automática dos registros para que os resultados possam ser facilmente generalizáveis.

\section{Referências}

Resnick, M., Maloney, J., Monroy-Hernandez, A., Rusk,N., Eastman, E., Brennan, K., Kafai, Y. (2009) "Scratch: Programming for All". Communications of the ACM, vol. $52, \mathrm{n}^{\mathrm{o}} 5$, pp. 31-33. 
V Congresso Brasileiro de Informática na Educação (CBIE 2016)

Anais dos Workshops do V Congresso Brasileiro de Informática na Educação (CBIE 2016)

Guzdial, M. (2009) "Education: Teaching Programming for everyone". Communications of the ACM, vol. 52, $\mathrm{n}^{\circ} 11, \mathrm{pp} .60-67$.

Kitchenham, B. A. (2007) "Guidelines for performing Systematic Literature Reviews in Software Engineering”. EAST' 12.

Pazinato, A. M. \& Teixeira, A. C. (2013). "O uso do software Scratch no desenvolvimento da aprendizagem e na interação construtivista dos alunos." Anais do XI Congresso Brasileiro de Ensino Superior a Distância, Belém/PA.

Wilson, A., Moffat, D. C. (2010). "Evaluating Scratch to introduce younger schoolchildren to programming". Proc. Of the Psychology of Programming Interest Group Workshop, Madrid/Espanha.

Calder, N. (2010). "Using Scratch: an integrated problem-solving approach to mathematical thinking." Australian Primary Mathematics Classroom, 15(4), 9-14.

Lamb, A., Johnson, L (2011). "Scratch: computer programming for $21^{\text {st }}$ century learners." Teacher Librarian. 38(4), 38-38, 75.

Wilson, A., Heiney, T., Connolly, T.M. (2013). "Using Scratch with primary school children: an evaluation of games constructed to gauge understanding of programming concepts.

Smith, A. C. (2010). "Dialando: tangible programming for the novice with Scratch, processing and Arduino. $6^{\text {th }}$ International Workshop on Technology for Innovation and Education in Developing Countries, Maputo, Mozambique, 21-23, 1-4.

Wing, J. (2006) “Computational Thinking”. Communications of the ACM, vol. 49, no. 3, pp.33-35.

Nunes, D. J. (2011) "Ciência da Computação na Educação Básica”. Jornal da Ciência. 09 de Setembro.

Monroy-Hernández, A., Resnick, M. (2008) "Empowering kids to create and share programmable media. Magazine: Interactions - Pencils before pixels: a primer in hand generated sketching. Volume 15 Issue 2, Pages 50-53.

Scaffidi, C., Chambers, C. (2012) "Skill progression demonstrated by users in the Scratch animation environment. International Journal of Human-Computer Interaction 28, 6 .

Dasgupta, S., Clementts, S., Idlbil, A., Willis-Ford, C., Resnick, M. (2014) "Extending Scratch: New Pathways into Programming." Visual Languages and Human-Centric Computing conference. Atlanta

Roque, R., Rusk, N., Resnick, M. (2016) "Supporting Diverse and Creative Collaboration in the Scratch Online Community". Mass Collaboration and Education, edited by U. Cress et al., pp. 241-156. Springer.

Resnick, M. (2007). "Learning from Scratch”. Microsoft Faculty Connection.

França, R. S., Silva, W. C., Amaral,. H. J. C. (2013) "Despertando o interesse pela ciência da computação: Práticas na educação básica”. Pages 282-286. 
V Congresso Brasileiro de Informática na Educação (CBIE 2016)

Anais dos Workshops do V Congresso Brasileiro de Informática na Educação (CBIE 2016)

CSTA - Computer Science Teacher Association. (2011) "CSTA K-12 Computer Science Standards". CSTA Standards Task Force. ACM - Association for Computing Machinery.

Wilson, A., Moffat, D. C. (2010) "Evaluating Scratch to introduce younger schoolchildren to programing". Pages 1-12.

Wang, X., Zhou, Z. (2011). "The research of situational teaching mode of programming in high school with Scratch." 6th IEEE Joint International Information Technology and Artificial Intelligence Conference (ITAIC), 2011, Chongqing, 488-492.

Harvey, B., Mönig, J. (2010). "Bringing 'No ceiling' to Scratch: can one language serve kids and computer scientists?" Constructionism 2010, Paris, 1-10. 